\title{
Unsteady natural convection in a triangular enclosure induced by absorption of radiation
}

\author{
By CHENGWANG LEI AND JOHN C. PATTERSON \\ School of Engineering, James Cook University, Townsville, Australia 4811
}

(Received 10 March 2001 and in revised form 10 August 2001)

The authors have previously reported a model experiment on the unsteady natural convection in a triangular domain induced by the absorption of solar radiation. This issue is reconsidered here both analytically and numerically. The present study consists of two parts: a scaling analysis and a numerical simulation. The scaling analysis for small bottom slopes reveals that a number of flow regimes are possible depending on the Rayleigh number and the relative value of certain non-dimensional parameters describing the flow. In a typical situation, the flow can be classified broadly into a conductive, a transitional or a convective regime determined merely by the Rayleigh number. Proper scales have been established to quantify the flow properties in each of these flow regimes. The numerical simulation has verified the scaling results.

\section{Introduction}

Natural convection driven by imposed horizontal temperature gradients finds application in many natural and geophysical systems, and consequently a vast literature exists on the various forms of forcing (internal or external), geometries and temporal conditions of the resultant flows (steady or unsteady). Much of the work has been in the context of regularly shaped containers and idealized boundary and initial conditions. Excellent reviews of the topic may be found in, for example, Ostrach (1988) and Hyun (1994).

In practice, the applications have neither idealized boundary or initial conditions, nor simple geometries. Frequently, the geometries are complex, the forcing is unsteady and the boundary conditions are more complex than a relatively simple application of an isothermal or isoflux thermal condition. One geometry that has attracted relatively little attention is that of a triangular enclosure, although interest has increased over the last two decades. The interest in heating by means of internal sources has been comparatively limited.

The previous studies in triangular geometries have been motivated by two main applications: the fluid dynamics and heat transfer in an attic space with an imposed temperature difference between the base and the sloping boundary (e.g. Poulikakos \& Bejan 1983a,b; Salmun 1995a,b; Asan \& Namli 2000); and the fluid dynamics of nearshore lake waters, reservoir sidearms or other shallow-water bodies with a sloping bottom (e.g. Horsch \& Stefan 1988; Horsch, Stefan \& Gavali 1994; Farrow $\&$ Patterson 1993a, $b, 1994)$. Although there are similarities between the two classes of problem, there are also many differences, some of which are fundamental.

In the attic case, the triangular domain is filled with air, which typically has a Prandtl number $\mathrm{Pr} \sim O(1)$, although Bejan \& Poulikakos (1982) also considered the case where the space was filled with a porous medium. The analysis of the attic 
space was for the case of imposed isothermal conditions on the horizontal bottom and sloping roof of the cavity. In the night-time configuration, the upper surface was cooler than the lower boundary (Poulikakos \& Bejan 1983a,b); during the day, the converse was assumed. There is obvious potential for a Bénard type instability to occur during the night-time phase.

In the geophysical application, the fluid is water, with $\operatorname{Pr} \sim O(10)$, and the forcing is primarily through the unequal absorption of incident solar radiation during the day or unequal surface cooling fluxes at night, both resulting from a varying depth. Put simply for the day-time case, in the shallow parts, the vertically averaged volumetric rate of absorption of a spatially uniform incident radiation flux is greater than in the deeper parts, resulting in relatively warm shallow regions when compared with the deep regions. A convectively driven circulation results, with a warm outflow along the surface. A similar but converse argument may be made for the night-time cooling condition. The attic and geophysical problems are, at first sight at least, separated by the different forms of forcing.

The night-time attic problem (actually motivated by the geophysical application) was re-examined by Salmun (1995a). He demonstrated by numerical analyses that, under some circumstances, the motion became unstable and a multi-cellular flow resulted. A stability analysis (Salmun 1995b), using the techniques developed by Farrow \& Patterson $(1993 b)$ further examined the stability of this Bénard-like problem. Farrow \& Patterson $(1993 a, 1994)$ used an asymptotic analysis based on the assumption of small bottom slope to find analytical solutions to two models for the geophysical problem. The first of these was a diurnal model in which it was assumed that all of the radiation was absorbed in the available depth, resulting in relatively high heating rates in the shallow part (Farrow \& Patterson 1993a). This model was an extension of the earlier scaling and numerical analysis of Patterson (1987). The second model, described in Farrow \& Patterson (1994), introduced a more realistic daytime heating model which took explicit account of the depth dependent absorption of radiation. More importantly, this model assumed that any residual radiation at the bottom was absorbed by the bottom and re-emitted as a heat flux. This flux was greater at the shallow end than at the deeper, and gave rise to a destabilizing temperature gradient at the bottom.

This latter model then is driven by both the internal buoyancy sources and a boundary flux, and therefore, to some extent, combines the principles of both the attic model and the initial exposition of the geophysical model. In this case, there are competing stability effects: the stabilizing effect of the stratification introduced by the absorption of solar radiation in the upper part of the water column and the destabilizing effect of the bottom-boundary heat flux. Although this problem is framed in the context of the near-shore transport question, it is clearly relevant to any buoyancy-driven flow in which these competing stability effects are present.

Lei, Patterson \& Lee $(2000 b)$ conducted for the first time a model experiment to visualize the convection process in a triangular cavity driven by this combination of forcing mechanisms. In that experiment, a triangular tank with a sloping and absorptive bottom was exposed to radiation from a theatre spotlamp. The flow was visualized using a shadowgraph technique, and temperatures were measured simultaneously at discrete locations along the sloping bottom. The experiment revealed many features regarding the transient flow development in a triangular domain that have not been reported previously in the literature. However, the existing experimental results do not allow a meaningful parametric investigation of this flow. In fact, only one set of the experiment series has been reported in Lei et al. (2000b), and thus it is not sufficient to draw a full picture of the flow. 
In this paper, the unsteady natural convection in a triangular domain induced by the absorption of radiation is investigated with a combined analytical and numerical procedure. First, a scaling analysis is carried out to quantify the flow, and then a numerical solution is provided to verify the scaling results. A similar procedure has been previously applied to natural convection in a differentially heated rectangular cavity (Patterson \& Imberger 1980), to a rectangular cavity containing a distribution of heat sources (Patterson 1984) and in an attic space (Poulikakos \& Bejan 1983a), and has proved to be successful. The numerical analysis confirms the scaling analysis, and is supported by the experimental results for the parameters available.

It is worth noting here that one of the imposed length scales of any problem involving the absorption of incident radiation is the attenuation length appropriate to the radiation and the fluid. Typically, in natural water bodies, this length scale is $O(1-2 \mathrm{~m})$, i.e. most of the radiation has been absorbed between the surface and this depth. This means that for regions deeper than the attenuation depth, the boundary flux will be small, and most of the incident radiation will be used in stratifying the upper part of the water column. Consequently, a shallow-water body with a sloping bottom may be separated into two regions: the first, a shallow region near the shore in which the forcing is dominated by the boundary flux mechanism described above; and the second deeper region in which the incident radiation serves only to stratify the upper part of the water body, with the motions being driven passively by the events of the first region. In this paper, we consider only the region affected by the bottom heat flux; that is, those parts for which the depth is less than the attenuation depth. A study of the interaction between these two regions is a more complex matter, and is beyond the scope of the present paper.

\section{Formulation and scaling}

Under consideration is a two-dimensional wedge of length $L$ and maximum depth $h$ with rigid non-slip boundaries at the bottom and end and an open boundary at the top (see figure 1). The sloping bottom is assumed to be black so that it absorbs any radiation reaching the bottom. The bottom slope, denoted by $A$, is defined as $A=\tan \theta=h / L$, where $\theta$ is the inclination angle of the bottom about the horizontal plane. The wedge is filled with water initially at rest and at temperature $T_{0}$. At time $t=0$, a surface radiation of intensity $I_{0}$ is initiated and thereafter maintained. When the surface radiation travels through a water column, the radiation intensity at a particular wavelength decreases with depth according to Beer's law (e.g. Rabl \& Nielsen 1975; Kirk 1986; Farrow \& Patterson 1994):

$$
I=I_{0} \mathrm{e}^{\eta y} \quad(y \leqslant 0),
$$

where $I$ is the radiation intensity at a given depth, $y$ the negative water depth, and $\eta$ the attenuation coefficient of water. Although the attenuation coefficient for water is strongly dependent on the wavelength of the radiation (Hale \& Querry 1973; Rabl \& Nielsen 1975) and the turbidity of water, in most limnological applications, it is often assumed that the absorption of radiation may be characterized by a single bulk attenuation coefficient, and that assumption is also followed here. It is seen from equation (1) that there will be only limited radiation reaching a water depth beyond $\eta^{-1}$. For the present analysis, it is assumed that the maximum water depth in the wedge is at most of the same order as the attenuation length scale of the radiation, i.e. $h<\eta^{-1}$. In other words, this study is concerned with shallow waters, in which a significant amount of the surface radiation penetrates all the way through the water column and reaches the bottom. The shallow-water assumption implies that the length of the domain is about the order of $1 /(A \eta)$ or less, i.e. $L<1 /(A \eta)$. 


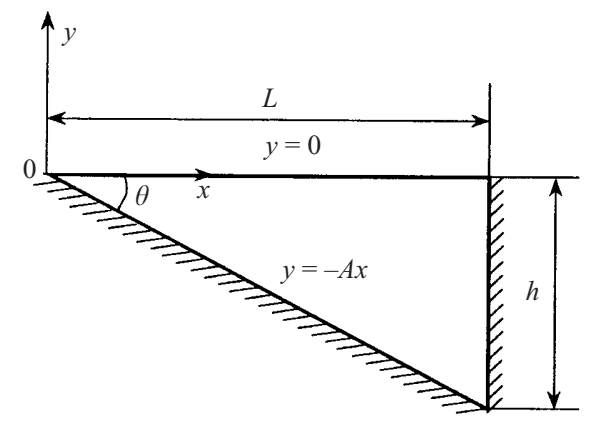

Figure 1. Geometry of the flow domain.

The water temperature in the enclosure increases as a consequence of the absorption of radiation. The subsequent flow and temperature changes within the wedge are then governed by the Navier-Stokes equations and energy equation with Boussinesq assumptions:

$$
\begin{gathered}
\frac{\partial u}{\partial t}+u \frac{\partial u}{\partial x}+v \frac{\partial u}{\partial y}=-\frac{1}{\rho_{0}} \frac{\partial p}{\partial x}+v \nabla^{2} u, \\
\frac{\partial v}{\partial t}+u \frac{\partial v}{\partial x}+v \frac{\partial v}{\partial y}=-\frac{1}{\rho_{0}} \frac{\partial p}{\partial y}+v \nabla^{2} v+g \beta\left(T-T_{0}\right), \\
\frac{\partial T}{\partial t}+u \frac{\partial T}{\partial x}+v \frac{\partial T}{\partial y}=k \nabla^{2} T+S(x, y, t), \\
\frac{\partial u}{\partial x}+\frac{\partial v}{\partial y}=0,
\end{gathered}
$$

where $x$ and $y$ are the horizontal and vertical coordinates originating from the tip (see figure 1), $u$ and $v$ are the horizontal and vertical components of velocity, $T$ is the temperature, $p$ is the pressure, $t$ is the time, $g$ is the acceleration due to gravity, $\beta, \rho_{0}, v$ and $k$ are the coefficient of thermal expansion, density, kinematic viscosity and thermal diffusivity for water at the reference temperature $\left(T_{0}\right)$, and $S(x, y, t)$ is an internal heating source which quantifies the absorption of radiation by the water. Since the absorption of radiation is characterized by a single bulk attenuation coefficient, the source term in equation (4), or the volumetric heating, is then given by (see also Farrow \& Patterson 1994)

$$
S=H_{0} \eta \mathrm{e}^{\eta y} \quad(y \leqslant 0),
$$

where $H_{0}$ is the surface heating intensity defined as $H_{0}=I_{0} /\left(\rho_{0} C_{p}\right)$, and $C_{p}$ is the specific heat for water at the reference temperature.

The internal heating source defined by equation (6) is a stabilizing force and is horizontally uniform. Therefore, the absorption of radiation by the water column itself will not generate a horizontal temperature gradient to drive a circulation in the tank. The principal driving force for the circulation comes from the heat exchange on the sloping bottom. In shallow waters, a significant amount of radiation will penetrate the water column and reach the bottom. For the present analysis, it is assumed that any residual radiation is fully absorbed by the black bottom, which then immediately releases this energy in the form of a boundary heat flux. Accordingly, the following 
boundary condition for the temperature is defined on the sloping bottom:

$$
\frac{\partial T}{\partial \hat{n}}=-\frac{1}{k} H_{0} \mathrm{e}^{-A \eta x} \quad(y=-A x),
$$

where $\hat{n}$ is the direction normal to the sloping bottom.

The rest of the boundary conditions for velocity and temperature are described as follows. On the sloping bottom $(y=-A x)$, rigid non-slip velocity conditions $(u=v=0)$ apply. The same velocity conditions apply to the endwall at $x=L$, which is also insulated (i.e. $\partial T / \partial x=0$ at $x=L)$. On the water surface $(y=0)$, it is assumed that any heat loss through the surface is small compared with the heat capture via absorption of radiation. In other words, it is assumed that the water surface is insulated (i.e. $\partial T / \partial y=0$ at $y=0$ ). It is also assumed that the water surface is stress-free $(\partial u / \partial y=0$ and $v=0)$. The stress-free surface condition is compatible with the experiment (Lei et al. 2000b) and the field condition in geophysical applications, and also matches the boundary condition used in Farrow \& Patterson (1994). Similar results are expected if a non-slip condition is specified on the top boundary. As can be seen from the subsequent scaling, the stress-free surface condition does not enter the scaling, and therefore the surface boundary condition should not alter the analytical results. The same is true for the scaling analysis in a rectangular cavity (Patterson \& Imberger 1980) where a non-slip condition is specified on the top boundary. Nevertheless, the non-slip boundary condition slightly modifies the numerical solution by creating an additional viscous layer near the surface. Apart from this modification, the overall flow features should remain the same as those under the stress-free condition. This has been demonstrated by Horsch \& Stefan (1988) in a numerical modelling of the corresponding surface cooling process in a triangular domain, where both stress-free and non-slip boundary conditions generated similar results.

\subsection{Development of the boundary-layer flow}

\subsubsection{Growth of the thermal boundary layer}

Before the surface radiation is initiated, there is no flow and no heat conduction in the enclosure. Apart from the region near the sloping bottom, initially there is no flow even after the surface radiation is applied since the absorption of penetrative radiation itself has no horizontal dependence. The water temperature in the tank increases owing to the absorption of the radiation by the water column. A balance between the unsteady and source terms in equation (4) yields a temperature growth scale

$$
T_{a} \sim H_{0} \eta \mathrm{e}^{\eta y} t \sim H_{0} \eta t .
$$

Note that $\mathrm{e}^{\eta y} \sim 1$ for $|y|<\eta^{-1}$. Similarly, $\mathrm{e}^{-A \eta x} \sim 1$ for $x<1 /(A \eta)$.

The above temperature scale applies to the entire flow domain except the bottom boundary region, where the water temperature increases owing to heat being conducted from the sloping bottom. From the boundary condition (7), the temperature growth scale in the thermal boundary layer is

$$
T_{b} \sim \frac{1}{k} H_{0} \delta_{T} \mathrm{e}^{-A \eta x} \sim \frac{1}{k} H_{0} \delta_{T},
$$

where $\delta_{T}$ is the thickness of the thermal boundary layer. A balance between the unsteady and diffusion terms in the energy equation, (4), yields a scale for the growth of the thickness of the thermal boundary layer

$$
\delta_{T} \sim(k t)^{1 / 2} .
$$


The experimental measurement has demonstrated the validity of the growth scale of the thickness of the thermal boundary (Lei et al. 2000b). Using (10), the temperature growth scale in the thermal boundary layer becomes

$$
T_{b} \sim \frac{H_{0}}{\delta_{T}} t \sim H_{0}\left(\frac{t}{k}\right)^{1 / 2} .
$$

For $t \ll h^{2} / k$ (the time scale for the bottom heat flux to diffuse over the entire depth), $\delta_{T} \ll \eta^{-1}$ (recall that $h<\eta^{-1}$ ), i.e. the temperature diffusion length scale is much smaller than the radiation penetrating scale. In natural water bodies, the typical observed value of the bulk attenuation coefficient is $O\left(1 \mathrm{~m}^{-1}\right)$ (Kirk 1986). A laboratory measurement in a water tank following the experiment of Lei et al. 2000b) gives an estimation of the bulk attenuation coefficient of $\eta=6.16 \mathrm{~m}^{-1}$, whereas the measured thickness of the thermal boundary layer was only up to a few millimetres (Lie et al. 2000b). Using the observed data, a comparison between (8) and (11) yields $T_{b} \gg T_{a}$. Thus, the heat conduction from the sloping bottom dominates the direct radiation absorption in the enclosure.

\subsubsection{Inception of the circulation}

The direct absorption of the radiation by the water column generates no horizontal temperature gradient, but causes vertical stratification in the wedge, which is a stabilizing process. Therefore, the temperature growth given by (8) does not cause any flow. However, the heat conduction from the sloping bottom to the thermal boundary layer produces a horizontal temperature gradient within the thermal layer in addition to a vertically adverse temperature gradient; thus, a horizontal pressure gradient is established which in turn drives a circulation in the cavity. There are two contributions to the horizontal pressure gradient, one is the horizontal component of the heat flux boundary condition at the sloping bottom, and the other is the variation of the temperature along the boundary layer. The former is approximately $A H_{0} \mathrm{e}^{-A \eta x} / k$ (derived from equation (7)), and the latter can be obtained from equation (9) by taking the partial derivative with respect to $x$, which gives $A H_{0} \eta \mathrm{e}^{-A \eta x}(t / k)^{1 / 2}$. A comparison between these two gradients suggests that the horizontal component of the heat flux boundary condition dominates up to $t \sim 1 /\left(k \eta^{2}\right)$. Therefore, it is expected that the isotherms in the thermal boundary layer are approximately parallel to the sloping bottom for small time. Since much of the flow development under concern will be complete within a time scale $t<h^{2} / k<1 /\left(k \eta^{2}\right)$, the contribution from the temperature variation along the boundary layer is excluded in the subsequent analysis.

It is assumed in the present study that the inclination angle of the bottom is very small, i.e. $A \ll 1$, which is typical in geophysical situations. This assumption admits the adoption of the thickness of the thermal boundary layer $\left(\delta_{T}\right)$ as an approximate vertical length scale. The small-slope assumption also implies that the horizontal component of the flow velocity approximately represents the flow velocity up the slope, while the vertical component of the flow velocity is negligible. Since $L<1 /(A \eta)$, i.e. the length of the domain is less than the horizontal length scale beyond which the variation of the thermal boundary condition becomes significant, the appropriate horizontal length scale for the thermal boundary layer is then $L=h / A$. A balance between the pressure gradient and buoyancy terms in the vertical momentum equation, (3), yields a scale for the pressure in the thermal boundary layer

$$
\frac{p}{\rho_{0}} \sim g \beta T_{b} \delta_{T} \sim g \beta H_{0} t .
$$


In the horizontal momentum equation, (2), the unsteady inertia term is $O(u / t)$, the advection term is $O\left(u^{2} / L\right)$, and the viscous term is $O\left(v u / \delta_{T}^{2}\right)$. The ratio of the advection term to the unsteady inertia term is $O(u t / L)$. For small enough time $t, u t<L$, and thus the advection term is insignificant when compared with the unsteady inertia term. Using (10), the ratio of the unsteady inertia term to the viscous term is $O\left(\mathrm{Pr}^{-1}\right)$, where $P r$ is the Prandtl number defined as

$$
\operatorname{Pr}=v / k .
$$

The present scaling analysis considers only flows with a Prandtl number greater than unity. For $\operatorname{Pr}>1$, the viscous term dominates the unsteady inertia term. Hence, the correct momentum balance in the horizontal direction is between the pressure gradient and the viscous terms, which then yields a velocity scale in the thermal boundary layer

$$
u \sim A \operatorname{PrGr}\left(\frac{t}{h^{2} / k}\right)^{2} \frac{k}{h},
$$

where $G r$ is the Grashof number defined by

$$
G r=\frac{g \beta H_{0} h^{4}}{v^{2} k} .
$$

Momentum is both advected away by the velocity, (14), and diffused into the core beyond the thickness $\delta_{T}$. The ratio of the advection to the diffusion of momentum over $\delta_{T}$ is $O\left(A^{2} G r\left(k t / h^{2}\right)^{3}\right)$. Therefore, for $t<t_{a}$, where $t_{a}$ is given by

$$
t_{a} \sim\left(\frac{1}{A^{2} G r}\right)^{1 / 3} \frac{h^{2}}{k},
$$

vorticity is diffused into the core, generating a secondary viscous layer of thickness

$$
\delta_{v} \sim(v t)^{1 / 2} .
$$

It is clear that $\delta_{v}$ is greater than $\delta_{T}$ for $\operatorname{Pr}>1$. Therefore, a double-layer structure appears in the region near the sloping bottom, with the inner thermal layer governed by a buoyancy-viscous balance and the outer viscous layer governed by an inertiaviscous balance.

\subsubsection{Steady state of the boundary layer}

While the thermal boundary layer is growing because of the heat being conducted into this layer from the sloping bottom, heat is also being convected away from the thermal boundary layer by the velocity, equation (14). The thermal layer will continue to grow until the heat conducted in from the sloping bottom is balanced by that convected away. In the early stage, the convective term of equation (4) is $O\left(u T_{b} / L\right)$ and the conduction term is $O\left(k T_{b} / \delta_{T}^{2}\right)$. Using (10) and (14), the balance between the convection and conduction terms yields a growth time scale for the thermal boundary layer

$$
t_{c} \sim\left(\frac{1}{A^{2} P r G r}\right)^{1 / 3} \frac{h^{2}}{k} .
$$

At $t \sim t_{c}$, the length, temperature and velocity scales for the boundary layer have become

$$
\delta_{T} \sim\left(\frac{1}{A^{2} P r G r}\right)^{1 / 6} h,
$$




$$
\begin{aligned}
T_{b} & \sim\left(\frac{1}{A^{2} \operatorname{Pr} G r}\right)^{1 / 6} \frac{H_{0} h}{k}, \\
u & \sim\left(A^{-1} \operatorname{Pr} G r\right)^{1 / 3} \frac{k}{h} .
\end{aligned}
$$

Thus, the inner thermal boundary layer grows until it has reached a thickness $\delta_{T}$ specified in (19) at time $t_{c}$. Since $t_{c}<t_{a}$ for $\operatorname{Pr}>1$, the diffusion of momentum out of the inner layer maintains the outer viscous layer, which has grown to a thickness

$$
\delta_{v} \sim\left(\frac{P r^{2}}{A^{2} G r}\right)^{1 / 6} h \sim \operatorname{Pr}^{1 / 2} \delta_{T} .
$$

Over the length scale $\delta_{v}$, the advection and diffusion of vorticity balance at time $t_{c}$. Therefore, $t_{c}$ is also the time scale for the steady state of the double-layer structure, in which an inner thermal layer governed by a balance of heat conduction and convection is contained by an outer viscous layer governed by a balance of advection and diffusion of vorticity.

If $\delta_{T} \ll h$, the thermal boundary layer is distinct, which requires

$$
\operatorname{Pr} G r>A^{-2},
$$

and if $\operatorname{PrGr}>A^{-2} \mathrm{Pr}^{3}$, the double-layer structure is distinct.

\subsection{Onset of the thermal layer instability}

Since the thermal boundary layer develops an adverse temperature gradient because of the heat being conducted from the bottom, this layer is potentially unstable. If the adverse temperature gradient exceeds a critical value, the instability will set in. Previous experiments and theoretical analyses (Chandrasekhar 1961; Drazin \& Reid 1981) have revealed that the stability of a fluid layer heated from below is characterized by a Rayleigh number, $R a$, which is defined as

$$
R a=\frac{g \beta d^{4}}{v k}\left|\frac{\mathrm{d} T}{\mathrm{~d} y}\right| \quad \text { or } \quad R a=\frac{g \beta \Delta T d^{3}}{v k},
$$

where $d$ is the depth of the fluid layer, $\Delta T$ the temperature difference between the upper and lower boundaries, and $|\mathrm{d} T / \mathrm{d} y|$ is the adverse temperature gradient maintained. Accordingly, a critical Rayleigh number exists, above which the instability will occur.

Kurzweg (1970) has demonstrated that the critical Rayleigh number at the onset of convective instability in an inclined layer is directly obtainable from that of a differentially heated horizontal layer. The relationship between the two critical Rayleigh numbers is simply given by (see Kurzweg 1970 and also Chen \& Pearlstein 1989; Daniels, Plapp \& Bodenschatz 2000)

$$
R a_{c}(\theta)=R a_{c}\left(0^{\circ}\right) / \cos \theta,
$$

where $\theta$ is the inclination angle of a fluid layer about the horizontal plane (see figure 1), and $R a_{c}(\theta)$ and $R a_{c}\left(0^{\circ}\right)$ are the critical Rayleigh numbers for the inclined and horizontal fluid layers, respectively. For brevity, $R a_{c}(\theta)$ will be replaced by $R a_{c}$ hereinafter. The critical Rayleigh number for a horizontal layer depends on the boundary configurations and heating conditions, and is readily available in the literature (Chandrasekhar 1961; Drazin \& Reid 1981). For instance, the RayleighBénard instability appears at $R a_{c}\left(0^{\circ}\right)=1101$ if the top fluid surface is free and the bottom surface is rigid, but at $R a_{c}\left(0^{\circ}\right)=1708$ if both the top and bottom surfaces are 
rigid. In the present case, the thermal boundary layer is bounded by a rigid surface at the bottom and a cold water layer on the top, which is equivalent to the free-rigid boundary configuration, for which $R a_{c}\left(0^{\circ}\right)=1101$. This value is used in equation (25) to estimate the critical Rayleigh number for the inclined thermal boundary layer in subsequent analyses and calculations.

It is necessary to mention that the above criteria for the onset of convective instability in a horizontal fluid layer were obtained for a fluid layer with constant temperatures on the bounding surfaces and a linear temperature profile across the depth of the layer. However, in the present case, a constant heat flux rather than a constant temperature is maintained on the lower surface of the thermal boundary layer, and the temperature distribution in the thermal layer exhibits strong nonlinearity as indicated experimentally (see Lei et al. 2000b). Sparrow, Goldstein \& Jonsson (1964) investigated the thermal instability of a horizontal fluid layer with different boundary conditions and nonlinear temperature profiles, and found that the critical Rayleigh number was smaller if a constant heat flux was applied on the boundary or a nonlinear temperature profile was specified. Since there is no definite data available for the present flow conditions, the criteria for constant temperature boundary conditions and linear temperature profiles are carried over to the present analysis. It will soon become clear that a slight variation of the critical Rayleigh number does not alter subsequent discussions and any conclusions drawn from them.

The local Rayleigh number in the thermal boundary layer is now calculated from (24), for which the depth of the fluid layer is $\delta_{T}$ and the temperature difference across this layer is $T_{b}$. Using (10) and (11), the local Rayleigh number is given by

$$
R a \sim \operatorname{Pr} G r\left(\frac{t}{h^{2} / k}\right)^{2} .
$$

If $R a \geqslant R a_{c}$, where $R a_{c}$ is calculated in (25), the instability will set in. From (26), a critical time scale for the onset of thermal-layer instability at given Grashof and Prandtl numbers is obtained

$$
t_{B} \sim\left(\frac{R a_{c}}{\operatorname{Pr} G r}\right)^{1 / 2} \frac{h^{2}}{k} .
$$

For $t<t_{B}$, the thermal boundary layer is stable, and for $t \geqslant t_{B}$, the instability will set in. Since the global Rayleigh number $R a$ is given by $R a=P r G r$, the scale (27) indicates that the critical time for the onset of convective instability in the thermal boundary layer is a linear function of the reciprocal of the square root of the Rayleigh number.

So far, we have obtained two important time scales for the thermal boundary layer; first, the time scale for the growth of the thermal boundary layer ( $t_{c}$ given by (18)), and secondly, the time scale for the onset of the convective instability $\left(t_{B}\right.$ given by (27)). The comparison of these two time scales is given by $t_{c} / t_{B} \sim\left(\operatorname{PrGr} / A^{4} R a_{c}^{3}\right)^{1 / 6}$. Therefore, for

$$
\operatorname{PrGr}>A^{4} R a_{c}^{3}
$$

$t_{c}>t_{B}$, and the convective instability will set in before the growth of the thermal boundary layer ceases. On the other hand, if $\operatorname{Pr} G r<A^{4} R a_{c}^{3}, t_{c}<t_{B}$, the thermal boundary layer will reach its steady state before the convective instability sets in. Since in this case the local Rayleigh number will reach its maximum value at the time $t_{c}$, the convective instability will not occur even if the surface radiation is maintained.

Moreover, a critical Grashof number for the onset of thermal layer instability at a 


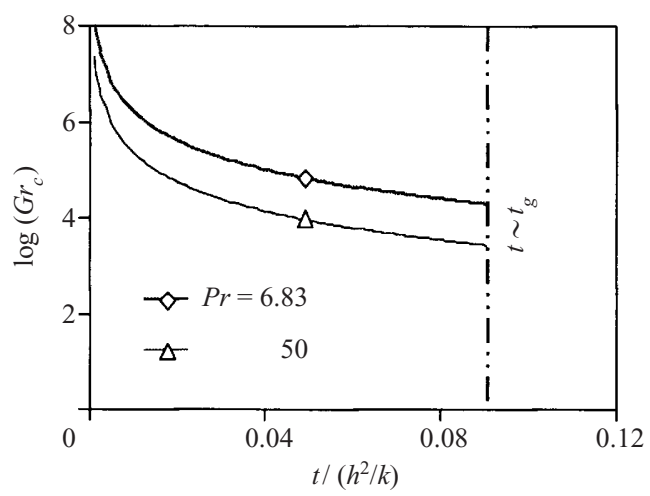

FIGURE 2. Dependence of the critical Grashof number on the time of heating.

given time and a fixed Prandtl number can also be obtained from (26)

$$
G r_{c} \sim \frac{R a_{c}}{\operatorname{Pr}}\left(\frac{h^{2} / k}{t}\right)^{2}
$$

For $G r<G r_{c}$, the thermal boundary layer is stable, whereas for $G r \geqslant G r_{c}$, the instability will set in. Clearly, the above criterion does not apply to $t \rightarrow \infty$ since there is a lower limit of the critical Grashof number for the convective instability to set in. This Grashof number can be obtained from (28), which requires $G r_{c}>A^{4} R a_{c}^{3} / P r$. Applying this criterion to (29) yields an upper limit of the time scale for the onset of convective instability, which is $t_{g} \sim\left(A^{2} R a_{c}\right)^{-1}\left(h^{2} / k\right)$. This time scale is identical to $t_{c}$ and $t_{B}$ for $\operatorname{PrGr} \sim A^{4} R a_{c}^{3}$.

The dependence of the critical Grashof number on the time of heating is plotted in figure 2. It is seen that as the heating time approaches zero, the critical Grashof number approaches infinity. The dependence of the critical Grashof number on the Prandtl number is also clearly demonstrated in figure 2, which shows that the instability is more prone to occur at large Prandtl numbers. The plot of the critical Grashof number at a fixed Prandtl number agrees qualitatively well with the lower bound of $G r_{c}$ given by Farrow \& Patterson (1993b), which was obtained from a linear stability analysis on the basis of an asymptotic solution. Both results indicate the same trend of the critical Grashof number with the heating time. However, the stability analysis of Farrow \& Patterson (1993b) covers a large-scale lake, and their result clearly shows the dependence of the critical Grashof number on the $x$-location. It is found that the most unstable region is around $x \sim 1 /(A \eta)$, and this region gradually moves toward deeper water as the heating time increases. The present scaling focuses on shallow waters, for which the $x$-dependence of the instability is neglected. Therefore, a quantitative comparison between this and the earlier studies is difficult.

\subsection{Possible flow regimes}

At this point, the natural convection flow in the shallow wedge subject to the surface radiation can be classified into different flow regimes depending on the Grashof and Prandtl numbers and the relative order of (23) and (28). The comparison between the scales (23) and (28) relies mostly on the bottom slope. There are two possibilities, i.e. $A^{-2}>A^{4} R a_{c}^{3}$ and $A^{-2}<A^{4} R a_{c}^{3}$, which are equivalent to the conditions $R a_{c}<A^{-2}$ and $R a_{c}>A^{-2}$. 
In the former case, i.e. $R a_{c}<A^{-2}$, the bottom slope is extremely small, and thus the boundary-layer flow is very similar to a horizontal layer heated from below. Consider first the situation of $\operatorname{PrGr}<A^{-2}$. The thermal boundary layer will grow to a scale of $O(h)$ at a time scale of $O\left(h^{2} / k\right)$ (obtained from (10)), at which the local Rayleigh number will also reach its maxima. From (26), the maximum local Rayleigh number is $\mathrm{O}(\mathrm{PrGr})$, which is of the same order as the global Rayleigh number. The criterion for the onset of the convective instability now becomes as simple as $\operatorname{Pr} G r>R a_{c}$. This criterion may also be obtained from (27) with the condition $t_{B}<h^{2} / k$ (i.e. the time scale for the onset of the convective instability is less than that for the temperature diffusion), and is consistent with the criterion for a horizontal fluid layer heated from below (Chandrasekhar 1961; Drazin \& Reid 1981). Condition (23) for the existence of a distinct thermal boundary layer automatically places the flow into the regime with the convective instability since $\operatorname{Pr} G r>A^{-2}>R a_{c}$ (note that $R a_{c}$ has replaced $A^{4} R a_{c}^{3}$ as the criterion for instability). In summary, the following flow regimes may be classified for the case $R a_{c}<A^{-2}$.

(i) $\operatorname{PrGr}<R a_{c}$. The flow is stable to the Rayleigh-Bénard instability. The thermal boundary layer grows steadily, and it encompasses the entire domain before convection becomes important. The heat transfer is dominated by conduction in this regime.

(ii) $\operatorname{Ra}_{c}<\operatorname{Pr} G r<A^{-2}$. The flow is fully unstable to the Rayleigh-Bénard instability, and the instability sets in at a time scale specified by (27). The subsequent heat transfer in the enclosure will be dominated by the secondary convection.

(iii) $\operatorname{PrGr}>A^{-2}$. A distinct thermal boundary layer develops along the sloping bottom, which is unstable to the Rayleigh-Bénard instability. The instability sets in at a time scale specified by (27). The heat transfer in this regime will be dominated by convection.

(iv) For sufficiently high Rayleigh numbers $(R a=P r G r)$, the thermal boundary layer becomes turbulent, similar to a horizontal layer heated from below (Burmeister 1993). The turbulent flow regime is out of the scope of the present analysis.

In the other case with $R a_{c}>A^{-2}$, the bottom slope may play a significant role. However, the present analysis still follows the assumption of $A \ll 1$. The relative order of the criteria (23) and (28) becomes $A^{-2}<A^{4} R a_{c}^{3}$, and thus the convective flow may be classified into the following flow regimes depending on the global Rayleigh number:

(i) $\operatorname{PrGr}<A^{-2}$. The flow is stable to the Rayleigh-Bénard instability. The thermal boundary layer grows steadily, and it encompasses the entire domain before convection becomes important. The heat transfer is dominated by conduction in this regime.

(ii) $A^{-2}<\operatorname{PrGr}<A^{4} R a_{c}^{3}$. Again, the flow is stable to the Rayleigh-Bénard instability. A distinct thermal boundary layer is established in the enclosure, and it reaches a steady state prior to the onset of the convective instability. For $\mathrm{PrGr}>$ $A^{-2} \mathrm{Pr}^{3}$, a double-layer structure with an inner thermal layer contained by an outer viscous layer is distinct. Both conduction and convection participate in the heat transfer in this regime.

(iii) $\operatorname{PrGr}>A^{4} R a_{c}^{3}$. The thermal boundary layer is still distinct in this regime, but it is unstable to the Rayleigh-Bénard instability. The convective instability sets in before the growth of the thermal boundary layer completes.

(iv) Similarly, there is also a regime at sufficiently high Rayleigh numbers, in which the thermal boundary layer becomes turbulent. This regime is not considered here.

It is noteworthy that the above classifications of different flow regimes apply to shallow regions, corresponding to the region of $x<1 /(A \eta)$ in Farrow \& Patterson (1994), which presents a zero-order asymptotic solution for a large-scale lake. For their 
asymptotic solution to be valid, it is required that $A^{2} G r$ must be small. Moreover, the nature of the zero-order asymptotic solution implies that only the effect of the vertical conduction is included in the solution whereas other effects such as the convection and horizontal conduction are excluded. Therefore, the asymptotic solution of Farrow \& Patterson (1994) only fits into the conductive flow regime up to the lower transitional regime of the present analysis, in which the vertical conduction is dominant.

The subsequent analysis will focus on the case with $R a_{c}>A^{-2}$ and consider only the laminar flow regimes. The flow development in the case with $R a_{c}<A^{-2}$ can be analysed with a similar procedure, and to a large extent, can be referred to the case of a horizontal layer heated from below, for which there is a large body of literature.

\subsection{Flow development in different regimes}

\subsubsection{In the regime $\operatorname{Pr} G r<A^{-2}<A^{4} R a_{c}^{3}$}

As noted previously, the thermal boundary layer eventually expands to the entire depth of the enclosure before a balance between heat conduction and convection is reached in this flow regime. The time scale for the diffusion of the boundary heat flux over the depth is

$$
t_{d} \sim \frac{h^{2}}{k} .
$$

At the time $t_{d}$, the temperature and velocity scales have become

$$
\begin{gathered}
T_{b} \sim \frac{H_{0} h}{k}, \\
u \sim \operatorname{APr} G r \frac{k}{h},
\end{gathered}
$$

and the temperature gradient over the depth of the domain is $O\left(T_{b} / h\right) \sim O\left(H_{0} / k\right)$, which is in an equilibrium with the heat flux condition applied on the sloping bottom. Therefore, no heat conduction over the depth will take place after the time $t_{d}$. Instead, the horizontal heat conduction will dominate owing to the existence of a horizontal temperature gradient as a consequence of the topographic effect. The time for the flow to reach a final steady state will be the temperature diffusion time scale over the length of the domain, which is

$$
t_{L} \sim \frac{L^{2}}{k} \sim A^{-2} \frac{h^{2}}{k} \sim A^{-2} t_{d} .
$$

This flow regime is classified as a conductive regime with conduction dominating the heat transfer in both transient and steady states. At the steady state, the temperature profile in the enclosure will become close to vertically uniform, and the horizontal heat transfer will be dominated by conduction.

\subsubsection{In the regime $A^{-2}<\operatorname{Pr} G r<A^{4} R a_{c}^{3}$}

In this regime, a stable and distinct thermal boundary layer develops along the sloping bottom. Since the boundary layer flow is moving up the slope, when it reaches the tip of the triangular domain, it has no other choice but to change its direction and discharge into the core region. The core region is initially isothermal. Therefore, the unheated fluid from the viscous layer discharges as a potential flow into the central part of the domain, whereas the heated fluid from the thermal layer travels across the top of the domain, and thus an upper intrusion layer is formed. The flux of the discharge of thermal boundary-layer flow is $Q_{H} \sim u \delta_{T}$. For $t<t_{c}, Q_{H}$ increases 
with time, while for $t \geqslant t_{c}, Q_{H}$ becomes constant. Invoking the principles of mass conservation, the volumetric flow rate of the intrusion flow must be the same as that of the thermal boundary layer flow, i.e.

$$
u_{i} \Delta \sim u \delta_{T},
$$

wher $u_{i}$ is the velocity of the intrusion flow, and $\Delta$ is the thickness of the intrusion layer.

For the intrusion flow, the appropriate horizontal length scale is the length of the domain $(L)$, and the vertical length scale is the thickness of the intrusion layer (4).Therefore, the inertia term of the intrusion flow is $O\left(u_{i} t\right)$, the advection term is $O\left(u_{i}^{2} / L\right)$, and the viscous term is $O\left(v u_{i} / \Delta^{2}\right)$. The intrusion flow is driven by a buoyancy-induced horizontal pressure gradient of $O\left(g \beta T_{b} \Delta / L\right)$. For $t<t_{c}$, the correct momentum balance within the intrusion layer is between the viscous term and the horizontal pressure gradient. Thus,

$$
v \frac{u_{i}}{\Delta^{2}} \sim \frac{g \beta T_{b} \Delta}{L} .
$$

Combining (34) and (35) and using (10), (11) and (14) yields the thickness and velocity scales of the intrusion flow as

$$
\begin{gathered}
\Delta \sim(k t)^{1 / 2}, \\
u_{i} \sim \operatorname{APrGr}\left(\frac{t}{h^{2} / k}\right)^{2} \frac{k}{h} .
\end{gathered}
$$

These two scales are identical to the corresponding scales of the thermal boundary layer. This indicates that the intrusion layer is growing on the same scales as the thermal boundary layer.

Now the relative scales of inertia, advection and viscous terms for the intrusion flow can be verified. The ratio of inertia to viscous terms is $O\left(\Delta^{2} / v t\right) \sim O\left(\mathrm{Pr}^{-1}\right)$, and thus for $\operatorname{Pr}>1$, the inertia term is insignificant when compared with the viscous term. The ratio of the advection to viscous terms is $O\left(u_{i} \Delta^{2} / v L\right)$. For $t<t_{c}, O\left(u_{i} \Delta^{2} / v L\right)<$ $O\left(\mathrm{Pr}^{-1}\right)<1$, and thus the advection term is also insignificant when compared with the viscous term. Therfore, the viscous term is indeed dominant for $t<t_{c}$.

The growth of the intrusion layer will complete if the heat conduction balances the convection. A balance between these two terms gives a growth time scale for the intrusion layer

$$
t_{i} \sim\left(\frac{1}{A^{2} \operatorname{Pr} G r}\right)^{1 / 3} \frac{h^{2}}{k} .
$$

This time scale is identical to the growth time scale for the thermal boundary layer. At the time $t_{i}$, the thickness and velocity of the intrusion layer have grown to

$$
\begin{aligned}
& \Delta \sim\left(\frac{1}{A^{2} \operatorname{Pr} G r}\right)^{1 / 6} h, \\
& u_{i} \sim\left(A^{-1} \operatorname{Pr} G r\right)^{1 / 3} \frac{k}{h} .
\end{aligned}
$$

Again, these scales are identical to those of the thermal boundary layer. Since both the thermal boundary layer and the upper intrusion layer reach their steady state at 
the same time scale, the convective flow in the enclosure also reaches its steady state at this time scale. At $t \sim t_{i}$, the intrusion layer has penetrated a distance of

$$
u_{i} t_{i} \sim \frac{h}{A}=L,
$$

which is equivalent to the length of the domain. This suggests that the approach to steady state of the convective flow is also approximately indicated by the arrival of the intrusion head at the end wall. At the steady state, the volumetric flow rate across a sectional plane is given by

$$
Q \sim u_{i} \Delta \sim\left(\frac{P r G r}{A^{4}}\right)^{1 / 6} k .
$$

\subsubsection{In the regime $\operatorname{PrGr}>A^{4} R a_{c}^{3}>A^{-2}$}

This flow regime is characterized by the presence of convective instability, which is due to the development of an adverse temperature gradient in the thermal boundary layer. The development of the boundary-layer flow in the early stage (for $t<t_{B}$ ) is quantified by the scales (10), (11) and (14). At the time $t_{B}$, which is prior to the completion of the boundary-layer development, the thermal boundary layer becomes unstable to the Rayleigh-Bénard instability. The pattern of the secondary convection depends on a number of factors, such as the Grashof number, the bottom slope and the boundary conditions, and is out of the scope of the present investigation. The subsequent flow development is complicated, and thus cannot be quantified by a simple scaling analysis. However, a qualitative description of the process is not impossible.

An immediate consequence of the onset of the convective instability is to cause heat loss from the thermal boundary layer and further destruction of this layer. The lost heat from the bottom layer then heats up the upper fluid layer, which in turn reduces the adverse temperature gradient in the lower layer and tends to suppress the secondary convection. As the surface radiation persists, the lower layer is heated continuously by heat conduction from the sloping bottom, and the adverse temperature gradient builds up again. At a certain stage, the lower layer again becomes unstable and the secondary convection begins. The previous process is repeated. The cyclic process happening in an unstable thermal boundary layer is described by Foster (1971) in the context of intermittent convection as follows: the formation of a thermal boundary layer by diffusion, the instability of this layer when it becomes sufficiently thick, the destruction of the layer by convection, the dying down of the convection and the reformation of the thermal boundary layer by diffusion. This process results in a fluctuation of both the temperature and velocity in the enclosure.

For relatively low Rayleigh numbers, the secondary motion occurs only in a transient stage. After some adjustment of the temperature structure in the enclosure owing to the secondary convection, the horizontal convection associating with the main circulation effectively balances the heat conduction from the sloping bottom. Therefore, a steady state with no secondary convection will be reached. However, the situation for high Rayleigh numbers is different. Despite the heat loss from the secondary convection, the excessive heating from the sloping bottom cannot be fully balanced by the primary convection, and it is sufficient to build up a critical temperature gradient in a short time, and thus maintain a secondary convection. Therefore, the steady state will be oscillatory for sufficiently high Rayleigh numbers. 


\section{Numerical procedures}

In order to test the validity of the previous scaling analysis and obtain better understanding of the flow features in different flow regimes, the convective flow in the triangular enclosure is solved numerically. For the convenience of numerical solution, the governing equations are first simplified with a quasi-steady state assumption, and then normalized with properly selected scales. Details of the numerical procedures are given below.

\subsection{Quasi-steady state simplification}

For the convective flow considered in this study, the water body in the enclosure is heated continuously by absorbing the radiation penetrating through the water column, and there is no heat loss through the boundaries. Therefore, the water temperature will keep increasing without a limit, and there will be no steady state in terms of temperature. However, with a constant surface radiation being applied, a quasi-steady state may be reached in which the temperature gradients and flow velocities become steady. In the quasi-steady state, the temperature increases at the same rate everywhere in the tank, and the net increase of the temperature is a function of time. Therefore, the temperature can be split into two components

$$
T=\bar{T}(t)+\tau(x, y, t),
$$

wher $\bar{T}(t)$ is the spatially averaged temperature which increases in time, and $\tau(x, y, t)$ is the spatial variation of temperature which has a steady-state spatial distribution after a transition. The average temperature $\bar{T}(t)$ can be obtained from a balance of energy entering from the surface and that absorbed by the water body, which gives

$$
\bar{T}(t)=\frac{2 H_{0}}{h} t .
$$

It is seen that the average temperature in the tank increases linearly with time.

Substituting (43) into (4) and using (6) and (44) yields an energy equation with respect to $\tau$

$$
\frac{\partial \tau}{\partial t}+u \frac{\partial \tau}{\partial x}+v \frac{\partial \tau}{\partial y}=k \nabla^{2} \tau+\left(H_{0} \eta \mathrm{e}^{\eta y}-\frac{2 H_{0}}{h}\right) .
$$

Since all boundary conditions for the temperature $T$ involve temperature gradients only and are independent of time, the same boundary conditions apply for $\tau$ as for $T$. Equation (45) is identical to (4) except that there is an extra term of heat sink on the right-hand side. This is interpreted as placing a uniformly distributed heat sink equal to $2 H_{0} / h$ across the tank while it is heated by the absorption of radiation entering from the surface. When the heat loss from the sink is balanced by the heat capture from the absorption of radiation, a steady state is reached for $\tau$, which corresponds to the quasi-steady state for $T$. In the following context, no distinction is made between the quasi-steady state for the temperature $T$ and the steady state for the temperature $\tau$, and $\tau$ in (45) will be denoted by $T$. The complete system equations now consist of (2), (3), (45) and (5).

\subsection{Normalization}

The quantities in the governing equations are normalized by the following scales:

Length scale: $x, y \sim h$; 
Time scale: $t \sim \frac{h^{2}}{k}$

Temperature scale: $\left(T-T_{0}\right) \sim \frac{H_{0} h}{k}$;

Velocity scale: $u, v \sim \frac{k}{h}$;

Pressure scale: $\frac{1}{\rho_{0}} \frac{\partial p}{\partial x}, \quad \frac{1}{\rho_{0}} \frac{\partial p}{\partial y} \sim \frac{g \beta H_{0} h}{k}$;

Attenuation coefficient scale: $\eta \sim \frac{1}{h}$.

Using the above scales for equations (2), (3), (45) and (5), a non-dimensional form of the governing equations is obtained

$$
\begin{gathered}
\frac{\partial u}{\partial t}+u \frac{\partial u}{\partial x}+v \frac{\partial u}{\partial y}=-\operatorname{Pr}^{2} G r \frac{\partial p}{\partial x}+\operatorname{Pr}^{2} u \\
\frac{\partial v}{\partial t}+u \frac{\partial v}{\partial x}+v \frac{\partial v}{\partial y}=-\operatorname{Pr}^{2} G r \frac{\partial p}{\partial y}+\operatorname{Pr}^{2} v+\operatorname{Pr}^{2} G r T \\
\frac{\partial T}{\partial t}+u \frac{\partial T}{\partial x}+v \frac{\partial T}{\partial y}=\nabla^{2} T+\left(\eta \mathrm{e}^{\eta y}-2\right), \\
\frac{\partial u}{\partial x}+\frac{\partial v}{\partial y}=0 .
\end{gathered}
$$

All the quantities in (46)-(49) are now dimensionless.

\subsection{Numerical schemes}

The governing equations in the non-dimensional form are solved using a finitedifference scheme. The velocity components and temperature are directly obtained from the Navier-Stokes equations and the energy equation, and the pressure is calculated from a pressure Poisson equation. By taking the divergence of the NavierStokes equations and applying the continuity condition, the following pressure Poisson equation is obtained

$$
\nabla^{2} p=-\frac{1}{P r^{2} G r}\left(u_{x}^{2}+2 u_{y} v_{x}+v_{y}^{2}+\frac{\partial \tilde{D}}{\partial t}\right)+T_{y},
$$

where $\tilde{D}=u_{x}+v_{y}$ is the residual of continuity. The subscripts $x$ and $y$ denote partial derivatives with respect to horizontal and vertical directions. The term $\partial \tilde{D} / \partial t$ is retained in (50) in order to eliminate the accumulation of numerical errors (see Lei et al. 2000a and references therein). The temperature equation, Navier-Stokes equations and the pressure Poisson equation are solved alternatively to obtain the temperature, velocity and pressure fields.

A coordinate transformation is applied to transfer the physical domain to a computational domain so that the system equations are solved on a curvilinear mesh, on which the mesh points can be easily distributed as required. Details of the coordinate transformation can be found in Lei et al. (2000a). Equations (46) to (50) are then discretized in the transformed computational domain which contains an orthogonal mesh. Standard second-order central differencing scheme is used for all spatial derivatives except the nonlinear terms in the momentum and energy equations. The nonlinear convective terms are approximated with a modified second-order upwind 
scheme proposed by Kawamura \& Kuwahara (1984):

$$
\begin{aligned}
\left(f \frac{\partial \phi}{\partial \xi}\right)_{i, j}= & f_{i, j}\left(-\phi_{i+2, j}+8 \phi_{i+1, j}-8 \phi_{i-1, j}+\phi_{i-2, j}\right) / 12 \Delta \xi \\
& +\left|f_{i, j}\right|\left(\phi_{i+2, j}-4 \phi_{i+1, j}+6 \phi_{i, j}-4 \phi_{i-1, j}+\phi_{i-2, j}\right) / 4 \Delta \xi,
\end{aligned}
$$

where $\phi$ denotes any scalar such as $u, v$ or $T$ in the governing equations, $f$ a spatially dependent coefficient, $\xi$ a coordinate in the computational domain, $\Delta \xi$ the grid spacing, and $i$ and $j$ are the sequential numbers of mesh points in two directions of the computational domain. The above differencing scheme actually has third-order accuracy in space.

The time integration for the velocity components and temperature is by a secondorder time accurate backward differencing scheme:

$$
\frac{\partial \phi}{\partial t}=\frac{3 \phi^{n+1}-4 \phi^{n}+\phi^{n-1}}{2 \Delta t},
$$

where $\Delta t$ is the time step. The correction term on the right-hand side of the pressure Poisson equation is also discretized with this scheme. The system equations are then solved implicitly with a successive over-relaxation (SOR) method by an iterative procedure.

The numerical procedures described above have been tested extensively and validated against several benchmarks. They have also been successfully applied to the modelling of different flows (e.g. Lei et al. 2000a and references therein). Therefore, these procedures are applied directly to the present convective flow.

\section{Results and discussion}

For the discussions of the numerical results, two parameters that have practical significance are calculated from the numerical data: the volumetric flow rate (or the strength of the circulation) and the horizontal heat transfer rate. These two parameters are defined in a two-dimensional domain as (Horsch et al. 1994):

$$
\begin{gathered}
Q(x)=\frac{1}{2} \int_{-A x}^{0}|u| \mathrm{d} y, \\
H(x)=\int_{-A x}^{0}\left(u T-\frac{\partial T}{\partial x}\right) \mathrm{d} y,
\end{gathered}
$$

where $Q(x)$ and $H(x)$ are, respectively, the volumetric flow rate and horizontal heat transfer rate across a vertical sectional plane at a given $x$ location. These two quantities have been normalized using the scales $Q \sim k$ and $H \sim I_{0} h$, respectively. It can be seen from the definition that the horizontal heat transfer rate incorporates the contributions of both convection and conduction. If the volumetric flow rate (53) and the horizontal heat transfer rate (54) are integrated along the horizontal direction as follows:

$$
\begin{aligned}
& Q=\frac{1}{L} \int_{0}^{L} Q(x) \mathrm{d} x, \\
& H=\frac{1}{L} \int_{0}^{L} H(x) \mathrm{d} x,
\end{aligned}
$$


an averaged volumetric flow rate $Q$ and an averaged heat transfer rate $H$ across the entire triangular domain are obtained.

Numerical solutions are now obtained with the aforementioned numerical procedures for a fixed bottom slope $A=0.1$. A number of combinations of Prandtl and Grashof numbers (see table 1), which spread over all three laminar regimes under the condition $R a_{c}>A^{-2}$, are calculated. It is noteworthy that the boundary between adjacent flow regimes is not definite since a scaling factor applies to each scale. All calculations are conducted in a triangular domain of a dimensionless length $L=10$ and a depth of $h=1$. To avoid singularity at the tip in the numerical simulations, the tip is cut off at $x=1.0$, and an extra rigid non-slip and adiabatic wall boundary is assumed near the tip. The heat sink term in equation (48) is adjusted accordingly for the new geometry. It is anticipated that the cutoff tip will not modify the flow significantly. An $81 \times 61$ non-uniform mesh is used for all the calculations. The dimensionless bulk attenuation coefficient of water is taken from the experiment of Lei et al. (2000b) and a subsequent measurement which gives $\eta=0.37$. As a check of the validity of numerical solutions, the convective flow is first calculated for the case of $G r=2.51 \times 10^{6}$ and $P r=6.83$, for which experimental data is available (Lei et al. $2000 \mathrm{~b}$ ). It is found that the numerical simulation has reproduced most of the flow features that was observed in the experiment (Lei \& Patterson 2001).

A mesh and time-step dependence test has also been conducted for the previous high-Rayleigh-number case by halving the grid resolution and time step simultaneously so that the CFL (Courant-Freidrich-Lewy) number remains the same. The time histories of the calculated mean flow rate with both coarse and fine meshes are plotted in figure 3. It is seen in this figure that both solutions indicate three stages of the flow development, an initial growth stage, a transition stage and a quasi-steady stage. In the initial growth stage, the two solutions follow each other closely. The transition stage is characterized by the onset of convective instability. The starting time of the convective instability (see $\S 4.2 .3$ for the determination of this time) predicted with both the coarse and fine meshes is 0.01 , and the time to reach a quasi-steady state is around 0.15 . In the quasi-steady state, both solutions show a similar pattern. The average flow rate at the quasi-steady state (averaged from $t=0.15$ to 0.25 ) obtained from the coarse and fine meshes are 39 and 40, respectively, and the variation is less than $3 \%$. However, the solutions of the coarse and fine meshes diverge significantly in the transition stage, in which the convective instability is dominant. This is because the formation of convective cells is extremely sensitive to the grid resolution. Since the purpose of the present study is to identify the major scales for the development of the flow rather than resolve the details of the convective instability, the discrepancy between the coarse and fine meshes is tolerated. In addition, the previous case has a Rayleigh number close to the higher end of the laminar flow regime considered in the present study, whereas most of the cases calculated here have a Rayleigh number well below this limit. It is expected that the numerical solution becomes less sensitive to the grid resolution at low Rayleigh numbers. Therefore, the coarse mesh is used for the subsequent calculations.

The typical flow features in different flow regimes will be discussed in $\S 4.1$, and selected scales will be validated in $\S 4.2$.

\subsection{Flow structures in different regimes}

\subsubsection{In the regime $\operatorname{Pr} G r<A^{-2}<A^{4} R a_{c}^{3}$}

Figure 4 presents the numerical results of a representative case in this low-Rayleighnumber regime with $\mathrm{Pr}=4, \mathrm{Gr}=12$ and $A=0.1$. The steady-state temperature 


\begin{tabular}{|c|c|c|c|}
\hline \multirow[b]{2}{*}{ Prandtl number, $P r$} & \multicolumn{3}{|c|}{ Grashof number, $G r$} \\
\hline & $\operatorname{PrGr}<A^{-2}$ & $A^{-2}<\operatorname{Pr} G r<A^{4} R a_{c}^{3}$ & $\operatorname{PrGr}>A^{4} R a_{c}^{3}$ \\
\hline 2 & 10 & $\begin{array}{l}2 \times 10^{2}, 5 \times 10^{2}, 2 \times 10^{3}, 5 \times 10^{3}, \\
\quad 10^{4}, 2 \times 10^{4}, 5 \times 10^{4}\end{array}$ & \\
\hline 4 & 12 & & \\
\hline 6.83 & 5 & $\begin{array}{l}5 \times 10^{2}, 1.03 \times 10^{3}, 2.87 \times 10^{3}, \\
\quad 6.45 \times 10^{3}\end{array}$ & $\begin{array}{l}2 \times 10^{4}, 7.17 \times 10^{4}, 1.61 \times 10^{5}, \\
\quad 6.45 \times 10^{5}, 2.51 \times 10^{6}, 1.03 \times 10^{7}, 10^{8}\end{array}$ \\
\hline 50 & & $5 \times 10^{2}, 10^{3}, 2.5 \times 10^{3}$ & $\begin{array}{l}7 \times 10^{3}, 2 \times 10^{4}, 7 \times 10^{4}, 1.5 \times 10^{5} \\
\quad 6 \times 10^{5}, 2.5 \times 10^{6}\end{array}$ \\
\hline 100 & & $1.03 \times 10^{3}$ & $\begin{array}{l}2.87 \times 10^{3}, 6.45 \times 10^{3}, 7.17 \times 10^{4} \\
\quad 1.61 \times 10^{5}, 6.45 \times 10^{5}, 2.51 \times 10^{6}\end{array}$ \\
\hline
\end{tabular}




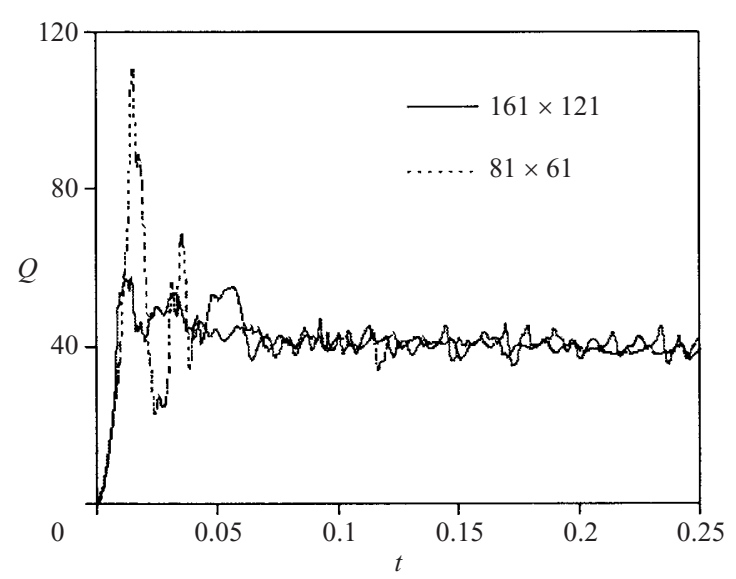

FIgURE 3. Time histories of the calculated mean flow rate for $\operatorname{Pr}=6.83, \mathrm{Gr}=2.51 \times 10^{6}$ and $A=0.1$ with two different meshes.

contours and streamlines are plotted in figures $4(a)$ and $4(b)$, respectively, and the time history of the integrated mean heat transfer rate (equation (56)) is given in figure $4(c)$. The contributions of conduction and convection to the horizontal heat transfer rate are presented separately in figure 4(c), which clearly indicates that this flow regime is completely dominated by conduction in both the transient and steady states. The contribution from convection is very close to zero throughout the calculation, and thus the two plots of the total and conductive heat transfer rates are indistinguishable. Since the thermal boundary layer eventually expands to the entire domain, the heat conduction within the thermal boundary layer creates a uniform temperature profile over the local depth. The approach to steady state consists merely of vertical isotherms moving out from the shallow end towards the deep end (figure $4 a$ ). The slight deflection of the isotherms from the vertical direction is due to the effect of the heat flux boundary condition. At the steady state, an approximately linear horizontal temperature gradient is established across the enclosure, which maintains an extremely weak circulation. The streamlines typically show a single closed cell structure (figure $4 b$ ). The dashed streamlines in figure $4(b)$ indicate a clockwise circulation in the enclosure.

\subsubsection{In the regime $A^{-2}<\operatorname{PrGr}<A^{4} R a_{c}^{3}$}

The numerical results of a representative case in this flow regime with $\operatorname{Pr}=2$, $G r=2 \times 10^{3}$ and $A=0.1$ are given in figure 5. Figures $5(a)$ and $5(b)$ present the steady state temperature contours and streamlines, respectively, and the time history of the calculated horizontal heat transfer rate is plotted in figure 5(c). In this medium-Rayleigh-number regime, convection becomes increasingly significant as the Rayleigh number increases, and both conduction and convection participate in the heat transfer. The isotherms are tilted in both the thermal boundary layer and the upper layer owing to the convective effect. However, vertical isotherms are still present in the region close to the shallow end (figure 5a), indicating that the heat transfer in the shallow region is dominated by conduction. Clearly, the circulation becomes much stronger in this flow regime (figure $5 b$ ), but the streamlines still show a single cell structure. The convective effect can be more or less important depending on the Rayleigh number. For the case presented here, the conductive and convective effects become equally important at the steady state (figure $5 c$ ). Note that the horizontal 
(a)

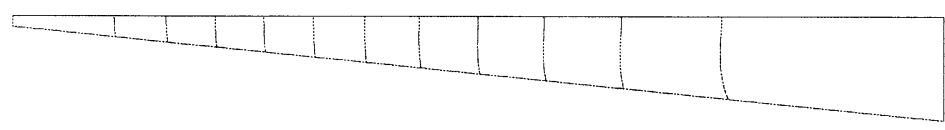

(b)

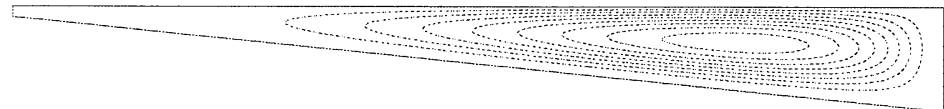

(c)

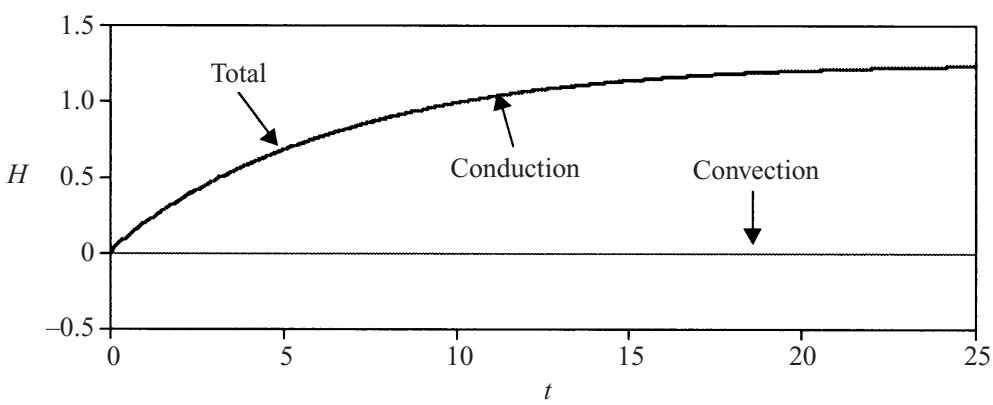

FIGURE 4. The numerical results for $\operatorname{Pr}=4, G r=12$ and $A=0.1$. (a) Steady-state temperature contours. The contour interval is 2. (b) Steady-state streamlines. The contour interval is 0.0192 . (c) Time history of the horizontal heat transfer rate.

(a)

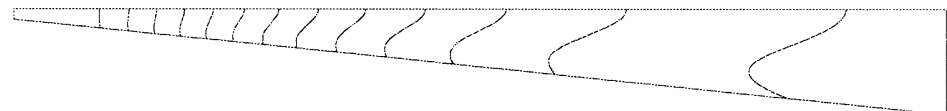

(b)

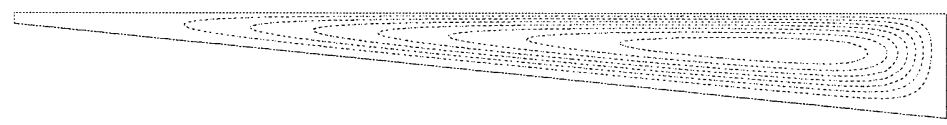

(c)

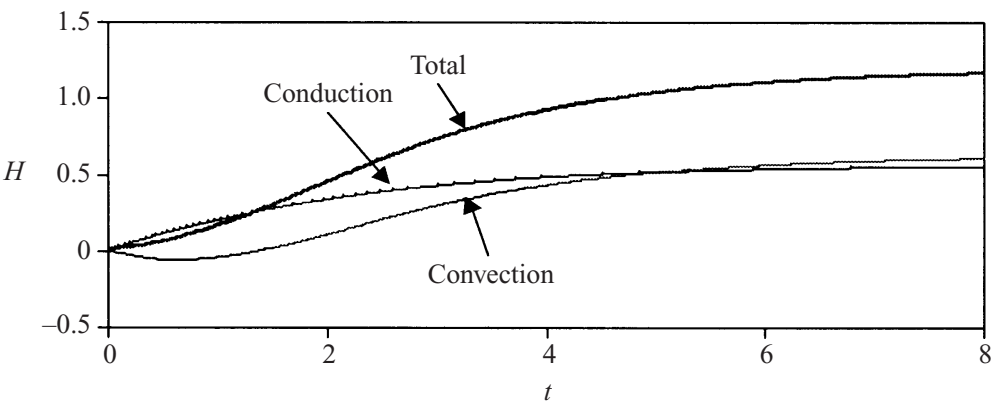

Figure 5. The numerical results for $\operatorname{Pr}=2, G r=2 \times 10^{3}$ and $A=0.1$. (a) Steady-state temperature contours. The contour interval is 1 . (b) Steady-state streamlines. The contour interval is 0.4. (c) Time history of the horizontal heat transfer rate.

convective heat transfer exhibits a distinct dip at the early stage in this flow regime (figure $5 c$ ). A similar dip can also be observed in the convective flow regime (figure $7 c$ ) which occurs on a shorter time scale. The existence of the dip indicates an imbalance between the strong up-slope flow of hot water in the boundary layer and the weak return flow of cool water in the bulk of the domain at the early stage. 
(a)

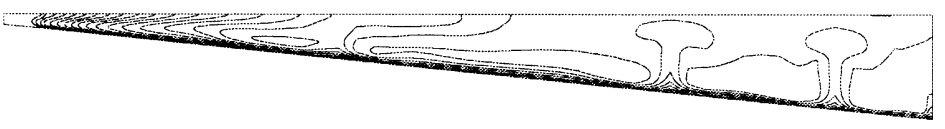

(b)

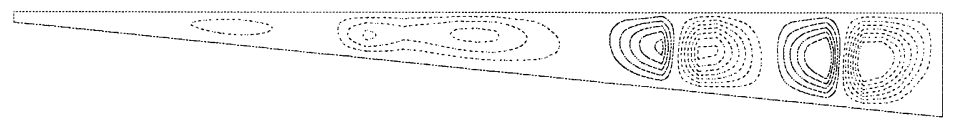

(c)

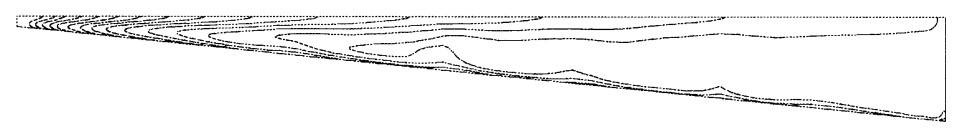

$(d)$

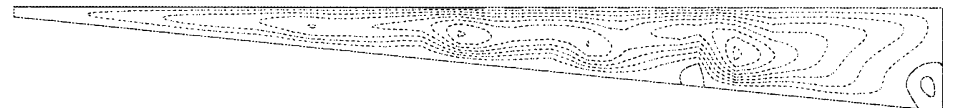

FiguRE 6. Instantaneous flow fields for $\operatorname{Pr}=6.83, G r=2.51 \times 10^{6}$ and $A=0.1$. (a) Temperature contours at transition stage $(t=0.03)$. The contour interval is 0.01 . (b) Streamlines at transition stage $(t=0.03)$. The contour interval is 20. (c) Temperature contours at quasi-steady stage $(t=0.2)$. The contour interval is 0.02 . (b) Streamlines at quasi-steady stage $(t=0.2)$. The contour interval is 10 .

(a)

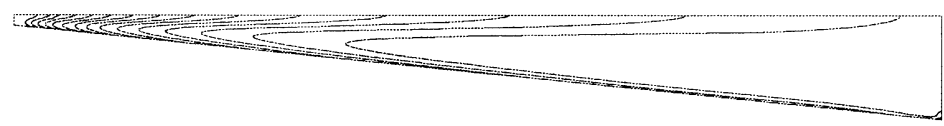

(b)

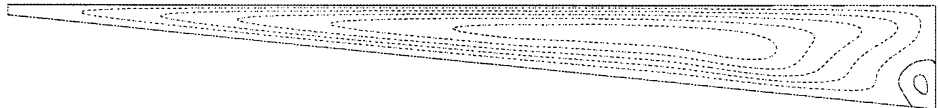

(c)

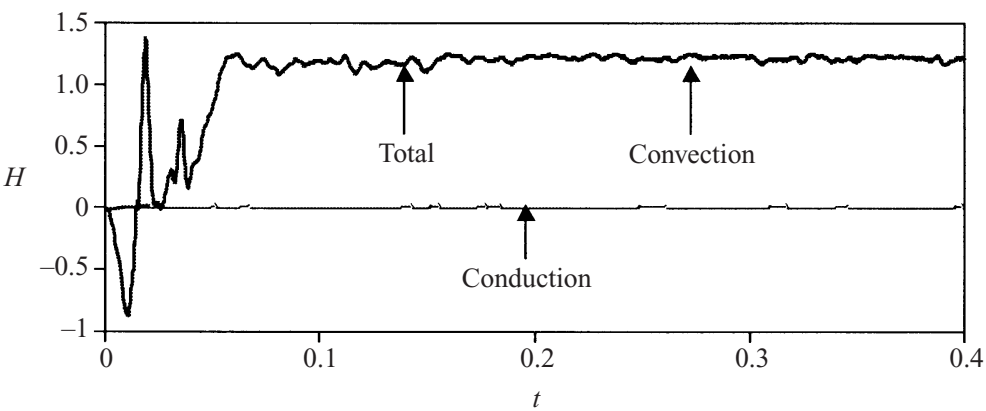

Figure 7. The numerical results for $\operatorname{Pr}=6.83, G r=2.51 \times 10^{6}$ and $A=0.1$. (a) Mean temperature contours at quasi-steady state (averaged for $t$ from 0.15 to 0.4 ). The contour interval is 0.02 . (b) Mean streamlines at quasi-steady state (averaged for $t$ from 0.15 to 0.4 ). The contour interval is 10. (c) Time history of the horizontal heat transfer rate.

\subsubsection{In the regime $\operatorname{Pr} G r>A^{4} R a_{c}^{3}>A^{-2}$}

This is the regime that is characterized by the presence of convective instability as a consequence of excessive heating in the thermal boundary layer. A representative case with $P r=6.83, G r=2.51 \times 10^{6}$ and $A=0.1$ is calculated to demonstrate the features of this flow regime. As noted previously, the flow development in this case occurs in three stages, an initial growth stage, a transition stage and a quasi-steady stage (see figure 3 and also figure $7 c$ ). The start of the transition stage is marked by the onset of the instability, which causes distinct fluctuations of flow properties such 
as the heat transfer rate (figure $7 c$ ). The quasi-steady state is reached when a steady constant mean value is achieved for all flow properties.

Figure 6 demonstrates the flow structures at the transition and quasi-steady stages. At the transition stage, the excessive heating from the sloping bottom results in the manifestation of the Rayleigh-Bénard instability in the form of rising thermals (figure $6 a$ ). Since initially the top fluid layer has a temperature much lower than that of the thermal boundary layer (recall that the present study is concerned with shallow waters with $h<\eta^{-1}$, and thus a significant amount of the radiation reaches the bottom), the rising thermals penetrate the entire depth of the enclosure and eventually reach the water surface. As a consequence, a cellular flow pattern is formed (figure $6 b$ ). The secondary convection accelerates the heat exchange between the top layer and the thermal boundary layer, and thus reduces the temperature gradient over the depth, which in turn weakens the secondary motion.

At the quasi-steady state, the intensity of the secondary convection has been reduced significantly. Since the temperature in the top layer has become comparably high compared with that in the thermal boundary layer at this stage, the secondary convection is now restricted within a thin layer above the thermal boundary layer (figure $6 c$ ). The weak secondary motion is not strong enough to break up the main circulation, and thus the main circulation contains only a single cell although multiple cores are visible owing to the weak effect of the secondary convection (figure $6 d$ ). The single-cell circulation could be due to the least stable mode occupying the entire domain as happens with fixed flux boundary conditions (Chapman \& Proctor 1980).

The structure of the mean flow at the quasi-steady stage (averaged from $t=0.15$ to $0.4)$ is presented in figures $7(a)$ and $7(b)$. It is seen in figure $7(a)$ that the temperature contours at the quasi-steady state consist of bent isotherms with the upper portion approximately parallel to the water surface and the lower portion nearly parallel to the sloping bottom, indicating the strong effect of convection. However, the isotherms become closer to vertical as the location moves from the deep region towards the tip, suggesting an increasing effect of conduction near the tip. The streamlines of the mean flow consist of two cells (figure $7 b$ ). The formation of the counterclockwise circulating cell near the lower corner of the deep end is due to the separation of the wall shear layer at this end, which does not appear at low Rayleigh numbers. As expected, the heat transfer in this high-Rayleigh-number regime is completely dominated by convection, which is clearly seen in figure $7(c)$. The two plots of the total and convective heat transfer rates are indistinguishable in figure 7(c) since the contribution from conduction is negligible in this case.

\subsubsection{Dependence of the steady-state heat transfer rate on the Rayleigh number}

The previous numerical results have demonstrated that the heat transfer in the triangular enclosure depends on the Rayleigh number. This is again shown in figure 8, where the total heat transfer rate and the separate contributions from convection and conduction at the steady/quasi-steady state are plotted against the Rayleigh number. The data in figure 8 are mostly obtained from the three lower Prandtl numbers given in table 1 . It can be seen in figure 8 that the total heat transfer rate at the steady or quasi-steady state is approximately constant $(\approx 1.2)$ over the entire calculated range of Rayleigh numbers, indicating that the overall heat transfer is proportional to the radiation intensity on the water surface (recall that the horizontal heat transfer rate is normalized by $I_{0} h$ ).

The contributions of conduction and convection to the total heat transfer are clearly demonstrated in figure 8 . At very low Rayleigh numbers, the flow is purely 


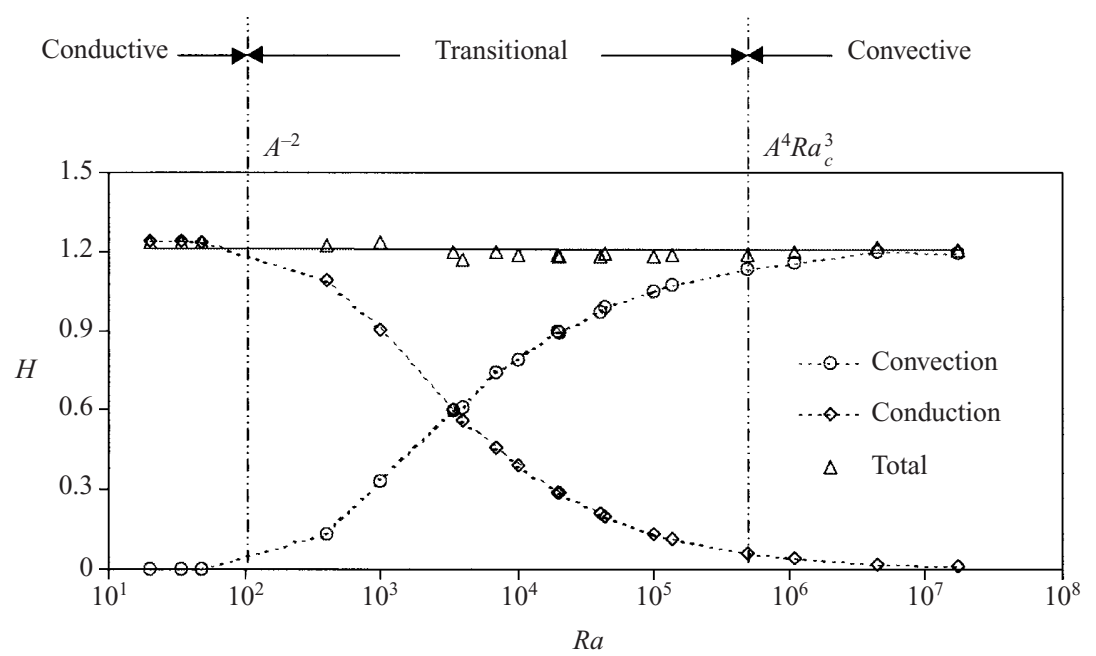

FIGURE 8. Dependence of the steady-state heat transfer rate on the Rayleigh number.

conductive, whereas at very high Rayleigh numbers, the flow is purely convective. At medium Rayleigh numbers, both conduction and convection contribute to the overall heat transfer. Therefore, the flow can be classified into three regimes: a conductive regime, a transition regime and a convective regime depending on the Rayleigh number. These three regimes are approximately separated by the criteria $A^{-2}$ and $A^{4} R a_{c}^{3}$, as discussed previously and marked in figure 8 .

\subsection{Validation of selected scales}

In the following context, selected property scales are validated against the numerical data in order to verify the validity of the previous scaling analysis.

\subsubsection{Maximum flow velocity}

The velocity scales for the transitional regime is considered here. The steady-state velocity in the thermal boundary layer, which is also the maximum flow velocity to be achieved in this layer, is governed by (21). The maximum flow velocity achievable in the upper intrusion layer is governed by (40). These two velocity scales are identical, suggesting that the maximum velocities in the thermal boundary layer and the intrusion layer are of the same order. The calculated maximum velocities in both layers are plotted against the dimensionless velocity scale in figure 9. Here, the numerical data are extracted from the steady-state velocity profile along a vertical line at $x=5.5$. It is clear that all the numerical data are represented well by the proposed velocity scales. The calculated slopes of the linear fit lines are 0.45 for the thermal boundary layer and 0.69 for the upper intrusion layer. Both slopes are of the order of unity. The numerical data presented in figure 9 actually span two flow regimes, the transitional and convective regimes. This indicates that the scales (21) and (40) for the maximum flow velocities in the thermal boundary layer and the intrusion layer, proposed for the transitional flow regime, are also applicable to the convective regime (for $\operatorname{PrGr}>A^{4} R a_{c}^{3}>A^{-2}$ ).

\subsubsection{Volumetric flow rate}

Again, the transitional flow regime is considered. The steady-state volumetric flow rate in this regime is governed by (42). The numerical data are plotted against the 

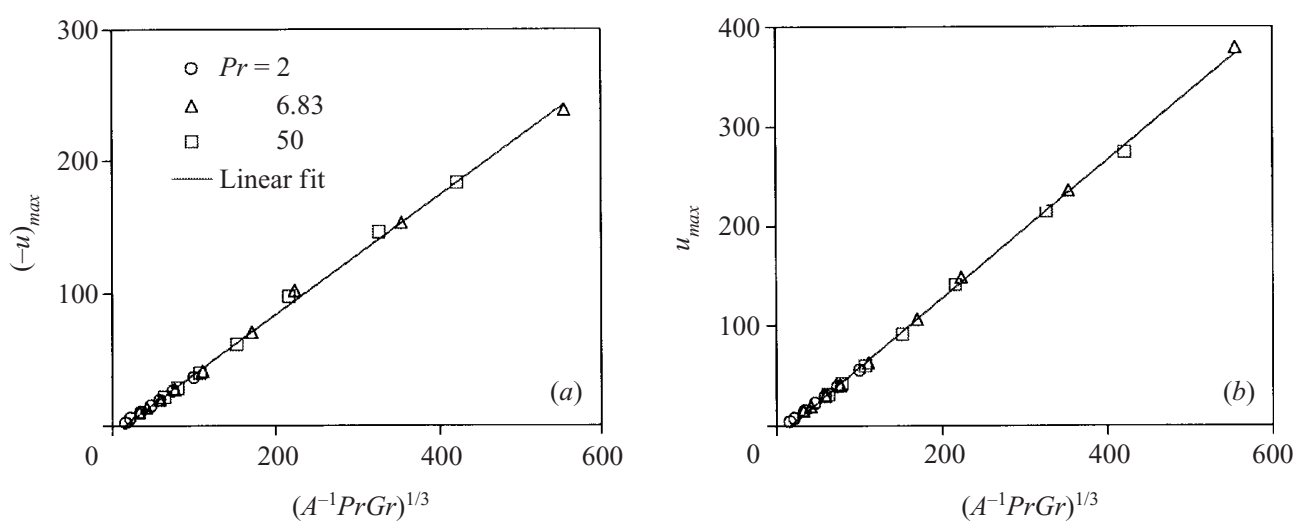

FIGURE 9. Maximum flow velocity extracted along a vertical line near the centre of the enclosure $(x=5.5)$. (a) Maximum negative horizontal component of velocity in the bottom boundary layer. (b) Maximum horizontal componenet of velocity in the upper layer.

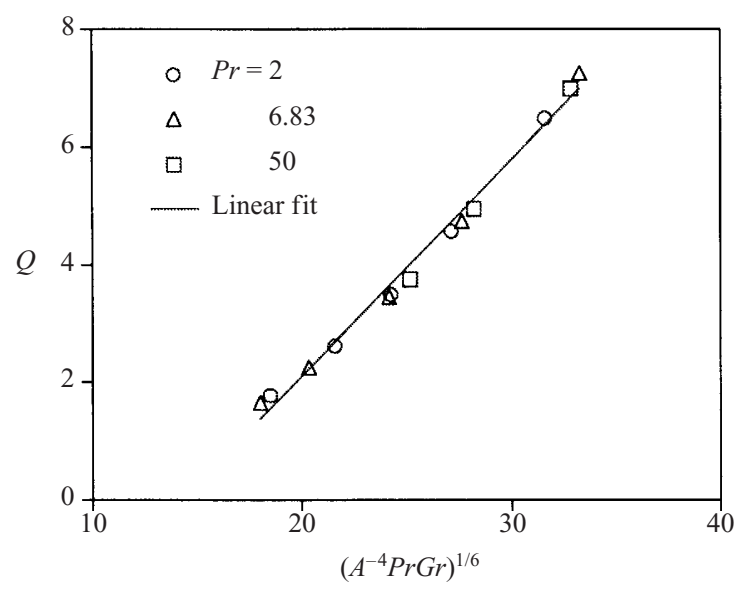

Figure 10. Integrated mean volumetric flow rate at the steady state.

dimensionless flow rate in figure 10. It is clear that the dependence of the calculated steady-state volumetric flow rate on the predicted non-dimensional flow rate is linear (the slope of the linear fit line is 0.37 ), and thus the scale (42) is representative for the steady-state flow rate in the transitional regime.

\subsubsection{Critical time for the onset of convective instability}

According to the scaling results in $\S 2.2$, the convective instability presents in the convective regime for $\operatorname{PrGr}>A^{4} R a_{c}^{3}$, and the critical time scale for the onset of the instability is $t_{B}$, given by (27). This scale is validated here, where the critical Rayleigh number $R a_{c}$ is calculated from equation (25) with $R a_{c}\left(0^{\circ}\right)=1101$. In the numerical simulation, the time $t_{B}$ for the onset of the convective instability is determined as the time when the first trough appears in the time history of the integrated mean heat transfer rate (see figures 3 and $7 c$ ). This is based on the fact that the onset of the convective instability results in a temperature drop in the thermal boundary layer and is always followed by subsequent fluctuations of flow properties owing to the secondary motion. With the integrated mean heat transfer rate used for the determination of $t_{B}$, the overall behaviour within the enclosure is concerned. The time 


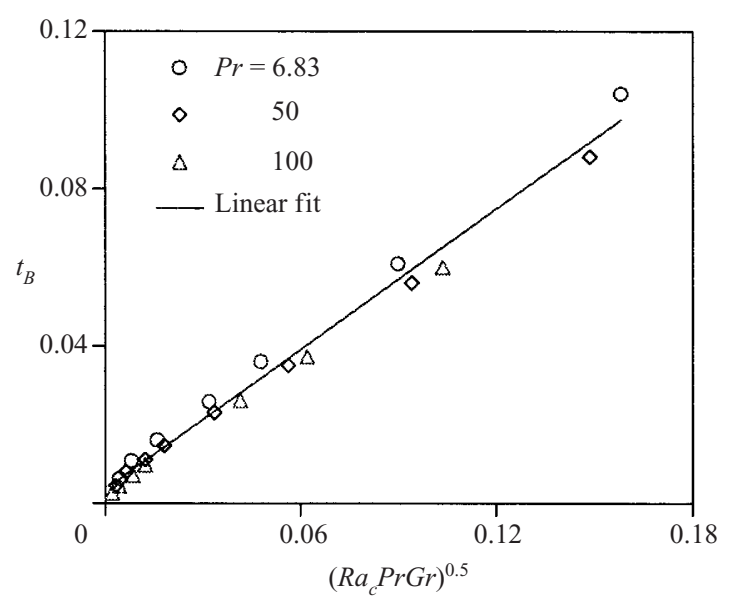

FIGURE 11. The calculated time for the onset of convective instability against the prediction of the scaling analysis. The discrete points are the numerical results, and the solid line is a linear fit of the numerical data.

$t_{B}$ may also be determined from the time plots of the horizontal heat transfer rates or temperatures at discrete locations. Since an inhomogeneous boundary condition is prescribed along the sloping bottom, the time $t_{B}$ determined from these discrete plots will be dependent on the horizontal location. However, it is expected that the result based on either method does not alter the conclusions drawn from the following validation.

The calculated time at the onset of the convective instability is plotted against the prediction in figure 11. The numerical data are mostly obtained at Rayleigh numbers in the convective regime, but also include some Rayleigh numbers at the upper end of the transitional regime. This plot clearly indicates a linear dependence between the calculated and predicted critical times. The slope of the linear fit line in figure 11 is 0.59 , which is of the order $O(1)$. Therefore, the critical time for the onset of the convective instability is represented by the scale (27).

\section{Conclusions}

The purpose of the present scaling analysis and numerical simulation is to identify possible flow regimes of the unsteady natural convection flow in a small-slope shallow wedge induced by the absorption of solar radiation, and establish relevant scales to quantify the flow properties in each regime. It is revealed that, in the case of $R a_{c}>A^{-2}$, this flow can be broadly classified into one of three regimes: a conductive regime, a transitional regime and a convective regime, depending on the Rayleigh number. The major features of each flow regime are summarized as follows:

(i) Conductive regime. For $R a<A^{-2}$, the flow is purely conductive. The thermal boundary layer grows to encompass the entire domain before convection becomes important. At the steady state, the energy from the surface radiation is uniformly distributed over the local depth, and thus an approximately linear horizontal temperature gradient is established across the enclosure. A weak circulation is maintained at the steady state.

(ii) Transitional regime. For $A^{-2}<\operatorname{Pr} G r<A^{4} R a_{c}^{3}$, both conduction and convection participate in the heat transfer. The convection is more or less important depending 
on the Rayleigh number. The transient flow development is described by the scales (10), (11) and (14). A steady state is reached when the conduction balances the convection in both the thermal boundary layer and the upper intrusion layer at time scales (18) and (38), which are identical. At the steady state, the thermal boundary layer is distinct, and the maximum flow velocity is given by (21) and (40).

(iii) Convective regime. For $\operatorname{PrGr}>A^{4} R a_{c}^{3}$, the flow becomes purely convective. The thermal boundary-layer development in the early stage is described by the scales (10), (11) and (14). At the time scale (27), the Rayleigh-Bénard convection sets in, and the subsequent flow development and approach to steady state become oscillatory. At a certain stage, a quasi-steady state is reached where both the primary convection and a secondary convection coexist. The mean flow at the quasi-steady state consists of a main circulation cell and a small bubble caused by the separation of the wall shear layer at the deep end. The numerical data indicate that the maximum flow velocity at the quasi-steady state is also quantified by (21) and (40).

Similarly three flow regimes are possible for other case with $R a_{c}<A^{-2}$, which shares some features of a horizontal fluid layer heated from below. The flow is stable and fully conductive if $\operatorname{Pr} G r<R a_{c}$, whereas it becomes fully unstable in the regime $\operatorname{Ra} a_{c}<\operatorname{PrGr}<A^{-2}$. Further increase of the Rayleigh number will result in a distinct thermal boundary layer developing along the sloping bottom, which is unstable to the Rayleigh-Bénard instability. The flow is fully convective in this high-Rayleigh-number regime.

At this point, the role of the critical Rayleigh number $\left(R a_{c}\right)$ becomes clear. It serves two purposes in the present analysis: dividing the flow regimes and affecting the time scale at the onset of the convective instability $\left(t_{B}\right)$. In this study, the critical Rayleigh number for the inclined thermal boundary layer is related to that of a horizontal layer heated from below (see equation (25)), and a critical value obtained with fixed temperature boundary conditions and a linear temperature distribution is adopted for the horizontal layer. It is understood that the conditions with constant temperatures on the boundaries and a linear distribution over the depth do not match the case considered here. However, the effect of this treatment is minimal. A slight variation of the critical Rayleigh number will move the boundaries that divide different flow regimes and change the slope of the linear-fit curve in figure 11. This is equivalent to the effect of a scaling factor for each scale. Therefore, the overall features of the natural convection flow will remain unchanged.

The scaling results may be verified by the experimental observations described in Lei et al. (2000b). The experiment was conducted in a triangular tank with a bottom slope of 0.1 , and the experimental Grashof number and Prandtl number were $2.51 \times 10^{6}$ and 6.83 , respectively. These parameters placed the flow in the unstable convective regime of $\operatorname{PrGr}>A^{4} R a_{c}^{3}>A^{-2}$. In this flow regime, a distinct thermal boundary layer is expected to grow along the sloping bottom. Using the flow parameters for the scale (27), the predicted time at the onset of instability is $202 \mathrm{~s}$. Since the numerical simulations have demonstrated that the maximum flow velocity in the thermal boundary layer is given by (21), the predicted flow velocity is $1.3 \mathrm{~mm} \mathrm{~s}^{-1}$ for the experiment. In the experiment, a thermal boundary layer was seen to grow on a scale of $(k t)^{0.5}$ with a scaling factor of 1.2, and a distinct thermal-layer instability was observed from about $60 \mathrm{~s}$ after the initiation of the surface radiation (Lei et al. $2000 \mathrm{~b}$ ). The intermittent occurrence of the instability created a wavy structure along the boundary layer, which was then carried by the main circulation up the slope. The observed flow velocity in the thermal boundary layer, indicated by the travelling speed of the wavy structure, was around $0.7 \mathrm{~mm} \mathrm{~s}^{-1}$ (not reported in Lei et al. 2000b). 
It can be seen that the scaling predictions are in good agreement with the observed data.

This research was funded by the Australian Research Council through an ARC Australian Postdoctoral Fellowship (F00104287). The authors are grateful to the anonymous reviewers who have provided valuable comments on an earlier version of this paper.

\section{REFERENCES}

AsAN, H. \& NAMLI, L. 2000 Laminar natural convection in a pitched roof of triangular cross-section: summer day boundary conditions. Energy Buildings 33, 69-73.

Bejan, A. \& Poulikakos, D. 1982 Natural convection in an attic-shaped space filled with porous material. Trans. ASME C: J. Heat Transfer 104, 241-247.

Burmeister, L. C. 1993 Convective Heat Transfer, 2nd edn. John Wiley.

Chandrasekhar, S. 1961 Hydrodynamic and Hydromagnetic Stability. Dover.

Chapman, C. J. \& Proctor, M. R. E. 1980 Nonlinear Rayleigh-Bénard convection between poorly conducting boundaries. J. Fluid Mech. 101, 759-782.

Chen, Y.-M. \& PeArlstein, A. J. 1989 Stability of free-convection flows of variable-viscosity fluids in vertical and inclined slots. J. Fluid Mech. 198, 513-541.

Daniels, K. E. Plapp, B. B. \& Bodenschatz, E. 2000 Pattern formation in inclined layer convection. Phys. Rev. Lett. 84, 5320-5323.

Drazin, P. G. \& ReID, W. H. 1981 Hydrodynamic Stability. Cambridge University Press.

FARrow, D. E. \& PATTERSON, J. C. $1993 a$ On the response of a reservoir sidearm to diurnal heating and cooling. J. Fluid Mech. 246, 143-161.

Farrow, D. E. \& Patterson, J. C. $1993 b$ On the stability of the near shore waters of a lake when subject to solar heating. Intl J. Heat Mass Transfer 36, 89-100.

Farrow, D. E. \& Patterson, J. C. 1994 The daytime circulation and temperature structure in a reservoir sidearm. Intl J. Heat Mass Transfer 37, 1957-1968.

Foster, T. D. 1971 Intermittent convection. Geophys. Fluid Dyn. 2, 201-217.

Hale, G. M. \& Querry, M. R. 1973 Optical constants of water in the $200 \mathrm{~nm}$ to $200 \mu \mathrm{m}$ wavelength region. Appl. Optics 12, 555-563.

Horsch, G. M. \& Stefan, H. G. 1988 Convective circulation in littoral water due to surface cooling. Limnol. Oceanogr. 33, 1068-1083.

Horsch, G. M., Stefan, H. G. \& Gavali, S. 1994 Numerical simulation of cooling-induced convective currents on a littoral slope. Intl J. Numer. Meth. Fluids 19, 105-134.

Hyun, J. M. 1994 Unsteady buoyant convection in an enclosure. Adv. Heat Transfer 24, 277-320.

Kawamura, T. \& Kuwahara, K. 1984 Computation of high Reynolds number flow around a circular cylinder with surface roughness. AIAA paper 84-0340.

KIRK, J. T. O. 1986 Optical limnology-a manifesto. In Limnology in Australia (ed. P. De Deckker \& W. D. Williams), pp. 33-62. CSIRO Australia and Dr W. Junk.

KURZWEG, U. H. 1970 Stability of natural convection within an inclined channel. Trans. ASME C: J. Heat Transfer 92, 190-191.

Lei, C., Cheng, L., Armfield, S. W. \& Kavanagh, K. 2000a Separation points and the near wake of a circular cylinder near a plane boundary. Comput. Fluid Dyn. J. 8, 521-535.

Lei, C. \& Patterson, J. C. 2001 A numerical simulation of the daytime natural convection in a reservoir sidearm. Proc. Intl Symp. on Advances in Computational Heat Transfer, Palm Cove. Australia, 20-25 May 2001, pp. 745-752.

Lei, C., Patterson, J. C. \& Lee, M. $2000 b$ Visualisation of natural convection in a reservoir sidearm subject to surface radiation. Proc. 7th Australasia Heat and Mass Transfer Conf. Townsville, Australia, 3-6 July 2000, pp. 201-208.

Ostrach, S. 1988 Natural convection in enclosures. Trans. ASME C: J. Heat Transfer 110, 1175 1190.

PAtTerson, J. C. 1984 Unsteady natural convection in a cavity with internal heating and cooling. J. Fluid Mech. 140, 135-151. 
Patterson, J. C. 1987 A model for convective motions in reservoir sidearm. Proc. 22nd Congress of IAHR, Laussane, 1987, pp. 68-73.

PAtterson, J. C. \& Imberger, J. 1980 Unsteady natural convection in a rectangular cavity. J. Fluid Mech. 100, 65-86.

Poulikakos, D. \& Bejan, A. $1983 a$ The fluid dynamics of an attic space. J. Fluid Mech. 131, 251-269.

Poulikakos, D. \& Bejan, A. $1983 b$ Natural convection experiments in a triangular enclosure. Trans. ASME C: J. Heat Transfer 105, 652-655.

Rabl, A. \& Nielsen, C. E. 1975 Solar ponds for space heating. Solar Energy 17, 1-12.

SAlmun, H. 1995 a Convection patterns in a triangular domain. Intl J. Heat Mass Transfer 18, $351-362$.

SAlmun, H. $1995 b$ The stability of a single-cell steady-state solution in a triangular enclosure. Intl J. Heat Mass Transfer 18, 363-369.

Sparrow, E. M., Goldstein, R. J. \& Jonsson, V. K. 1964 Thermal instability in a horizontal fluid layer: effect of boundary conditions and non-linear temperature profile. J. Fluid Mech. 18, $513-528$. 